\title{
Application of TALYS code for Calculation of Fission Cross Section and Fission Yield of Several Heavy Nuclides
}

\author{
Yuda S. Perkasa, Rizal Kurniadi, and Aabdul Waris* \\ Nuclear Physics and Biophysics Research Division \\ Faculty of Mathematics and Natural Sciences \\ Institut Teknologi Bandung \\ *e-mail: awaris@fi.itb.ac.id
}

\begin{abstract}
Nuclear data evaluation for fission cross section and fission yield had been performed by many investigators using different models of approximation theoretically. These models are encapsulated and implemented into computer codes to perform more robust nuclear reaction data calculations. TALYS is one of most successful nuclear reaction codes that used to determine fission cross section and fission yield.

In this paper, TALYS code was used to calculate some fission reaction including Am-241 (n,f), Th-232 (n,f), and U$235(n, f)$. These calculations are performed using different set of reaction mechanism and optical model parameter adjustment, such as fission barrier parameter, level density parameter, transmission mechanism, and so on. Reaction mechanism and parameter adjustment are selected based on reaction characteristics to obtain more accurate and reasonable result. The accuracy of calculation result are heavily depend on the reaction mechanism selection and parameter adjustment. All obtained results have been compared with ENDF nuclear data library.
\end{abstract}

Keywords: Fission cross section, Fission yield, TALYS code, Optical model, ENDF

\section{Introduction}

TALYS was born from idea to encapsulate all nuclear reaction model calculation into single software code that could provide complete and accurate nuclear reaction simulation from $1 \mathrm{KeV}-200 \mathrm{MeV}$ energy range ${ }^{1}$. Nuclear reaction type that provided by TALYS are nuclear reaction that involved neutron, proton, deuteron, photon, triton, He-3 and alpha particles as projectile and element of mass 12 amu and heavier as target.

Calculation results obtained from TALYS depend on fine input parameter tuning based on experimental value using curve fitting. At this point, experimental data play important role to achieve better agreement with experiment.

Besides TALYS there are other nuclear reaction codes that used extensively for academic purposes or even creation of nuclear data library. For examples: GNASH, ALICE, STAPRE, and EMPIRE. Each one of them has very specific different features. The specific features of TALYS are as follows:

- Exact implementation of latest nuclear model for direct, compound, pre-equilibrium and fission reaction,

- Smooth and continuous description of reaction mechanism over wide energy range $(0.001-$ $200 \mathrm{MeV})$ and wide mass number range (12 < A $<339$ ),

- Integrated optical Model and Coupled Channels calculation (ECIS-06 code),

- Total and partial cross section, energy spectra, angular distribution, double-differential spectra and recoils,
- Photon production cross section for discrete and continue distribution,

- Excitation functions for residual nuclide production, including isomeric cross section,

- Exact modeling for exclusive channel cross section,

- Calculation parameter using RIPL library,

- Various width fluctuation models for binary compound reaction,

- Using different level density models,

- Fission calculation using various models.

\section{A Brief Theoretical Overview}

\subsection{Transmission coefficient}

TALYS offer many options for fission model. The default model uses implemented transition hypothesis from Bohr and Hill-Wheeler expression. Fission model that used in TALYS yields concept of transmission coefficient from Hauser-Fesbach Model.

Total fission transmission coefficient for nucleus with excitation energy $E_{x}$, spin $J$, and parity $\Pi$ reads :

$$
\begin{array}{r}
T_{f}^{J \Pi}\left(E_{x}\right)=\sum_{i} T_{f}\left(E_{x}, \varepsilon_{i}\right) f(i, J, \Pi) \\
\quad+\int_{E_{t h}}^{E_{x}} \rho(\varepsilon, J, \Pi) T_{f}\left(E_{x}, \varepsilon\right) d \varepsilon
\end{array}
$$

Where,

$$
T_{f}\left(E_{x}, \varepsilon_{i}\right)=\frac{1}{1+\exp \left[-2 \pi \frac{\left(E_{x}-B_{f}-\varepsilon_{i}\right)}{\hbar \omega_{f}}\right]}
$$


is transmission coefficient for single barrier, $f(i, J, \Pi)=1$ if the spin and parity of the transition state equal that of the compound nucleus and 0 otherwise, and $\rho(\varepsilon, J, \Pi)$ is level density of fission channels. For double humped barrier, one introduces the effective transmission coefficients.

$$
T_{\text {eff }}=\frac{T_{A} T_{B}}{T_{A}+T_{B}},
$$

where $T_{A}$ and $T_{B}$ is transmission coefficient for barrier $A$ and $B$ and calculated using (1). Effective transmission coefficient for triple humped barrier reads

$$
T_{\text {eff }}=\frac{T_{A} T_{B} T_{C}}{T_{A} T_{B}+T_{A} T_{C}+T_{B} T_{C}}
$$

Transmission coefficients in TALYS also can be calculated using WKB approximation.

\subsection{Fission barrier parameter}

Transmission coefficients values have very important dependencies with fission barrier parameter. TALYS has provided several options for describing fission barrier parameter, namely:

- Experimental parameter barrier based on a fit with experimental data, compiled by $\mathrm{V}$. Maslov.

- Mamdouth parameter barrier contain set of double-humped fission barrier heights derived from Extended Thomas-Fermi plus Strutinsky Integral calculation ${ }^{6}$.

- Rotating-Finite-Range Model (RFRM) by Sierk is used to determine single-humped fission barrier heights.

- Rotating-Liquid-Drop Model (RLDM) by Cohen et. al.

Fission barrier dependent on angular momentum is discarded.

\subsection{Level densities}

Effective level densities had no explicit dependencies with nuclear collective effect. Several models can be used for calculating the level density parameter. Brief explanations of them are as follows.

1. Fermi Gas Model

This model assuming that the single particle states which construct the excited levels of the nucleus are equally spaced and that collective level are absent.

If it is assumed that the projections of the total angular momentum are randomly coupled, it can be derived (2) that the Fermi gas level density is defined as:

$$
\begin{aligned}
& \rho_{F}\left(E_{\chi}, J, \Pi\right)=\frac{1}{2} \frac{2 J+1}{2 \sqrt{2 \pi} \sigma^{3}} \\
& \exp \left[-\frac{(J+1 / 2)^{2}}{2 \sigma^{2}}\right] \frac{\sqrt{\pi}}{12} \frac{\exp [2 \sqrt{a U}]}{a^{1 / 4} U^{5 / 4}}
\end{aligned}
$$

Factor $\sigma^{2}$ is the spin cut-off parameter, which represents the width of angular momentum distribution. Level density parameter $a$ determined using Ignatyuk Formalism (3).

2. Constant Temperature Model

This model divides energy range into two parts, low energy part from $0 \mathrm{MeV}$ to the matching energy $E_{M}$ where constant temperature law applied, and high energy part above $E_{M}$ where Fermi Gas Model applied.

$$
\begin{aligned}
\rho^{\text {tot }}\left(E_{x}\right) & =\rho_{T}^{\text {tot }}\left(E_{x}\right), \quad & E_{x} \leq E_{M} \\
& =\rho_{F}^{\text {tot }}\left(E_{x}\right), \quad & E_{x} \geq E_{M}
\end{aligned}
$$

and for level density

$$
\begin{aligned}
\rho\left(E_{\chi}, J, \Pi\right) & =\frac{1}{2} R_{F}\left(E_{x}, J\right) \rho_{T}^{\text {tot }}\left(E_{x}\right), & & E_{\chi} \leq E_{M} \\
& =\rho_{F}\left(E_{x}, J, \Pi\right), & & E_{x} \geq E_{M}
\end{aligned}
$$

where

$$
R_{F}\left(E_{x}, J\right)=\frac{2 J+1}{2 \sigma^{2}} \exp \left[-\frac{(J+1 / 2)^{2}}{2 \sigma^{2}}\right]
$$

is Fermi gas spin distribution.

3. Back-Shifted Fermi Gas Model

In this model, Fermi gas expression is used in all energy range. As a consequence, pairing energy parameter should be adjustable.

Total level density reads :

$\rho_{F}^{\text {tot }}\left(E_{x}\right)=\frac{1}{\sqrt{2 \pi} \sigma} \frac{\sqrt{\pi}}{12} \frac{\exp [2 \sqrt{a U}]}{a^{1 / 4} U^{5 / 4}}$

and for level density :

$$
\begin{aligned}
& \rho_{F}\left(E_{X}, J, \Pi\right)=\frac{1}{2} \frac{2 J+1}{2 \sqrt{2 \pi} \sigma^{3}} \\
& \exp \left[-\frac{(J+1 / 2)^{2}}{2 \sigma^{2}}\right] \frac{\sqrt{\pi}}{12} \frac{\exp [2 \sqrt{a U}]}{a^{1 / 2} U^{5 / 4}}
\end{aligned}
$$

where :

$U=E_{x}-\Delta^{B F M}$

with pairing energy shift :

$\Delta^{B F M}=\chi \frac{12}{\sqrt{A}}+\delta$

4. Generalized Superfluid Model (GSM)

This Model takes superconductive pairing correlation into account based on BardeenCooper-Schrieffer theory. Level density for this model is :

$\rho_{G S M}\left(E_{\chi}, J, \Pi\right)=\frac{1}{2} R_{F}\left(E_{\chi}, J\right) \rho_{G S M}^{\text {tot }}\left(E_{\chi}\right)$

where total level density expressed as :

$\rho_{G S M}^{\text {tot }}\left(E_{\chi}\right)=\frac{1}{\sqrt{2 \pi} \sigma} \frac{\sqrt{\pi}}{12} \frac{\exp [2 \sqrt{a U}]}{a^{1 / 4} U^{5 / 4}}$ 


\subsection{Mass yield distribution}

Mass yield distribution in TALYS is determined using modified RNRM (Random Neck Rupture) Model from U.Brosa. This modified model is used to calculate the properties of fission fragment at higher temperature than zero one. RNRM model assumed that rupture process when fission takes place is a random process at different scission point at neck. This assumption yields Lawrencian shape that lead to the fission yield of product nuclide.

At the fission point, there are three possible modes or fission path that belong to fissioning nuclide, that is Super Long (SL), Standard I (ST I), and Standard II (ST II). All this modes is manifestation of the fission barrier shape and precision shape as function of temperature

Fission mass distribution is defined as :

$$
\begin{aligned}
& Y\left(A_{F F} ; Z_{F S}, A_{F S}, E_{x}\right) \\
& =\sum_{F M=S L, S T I, S T I I} W_{F M}\left(Z_{F S}, A_{F S}, E_{X}\right) Y_{F M}\left(A_{F F} ; Z_{F S}, A_{F S}, E_{\chi}\right)
\end{aligned}
$$

where $W_{F M}\left(Z_{F S}, A_{F S}, E_{x}\right)$ is weight of corresponding fission mode and $W_{F M}\left(A_{F F} ; Z_{F S}, A_{F S}, E_{x}\right)$ is corresponding mass distribution. Fission weight for SL mode defined as :

$$
W_{S L}\left(Z_{F S}, A_{F S}, E_{x}\right)=\frac{T_{f, S L}^{B}}{T_{f, S L}^{B}+T_{f, S T 1}^{B}+T_{f, S T I I}^{B}},
$$

while equivalent formulas hold for ST I and ST II modes.

The rupture probability at neck is proportional to the Boltzmann factor which depends on Lawrencian shape.

$$
y\left(A_{F F}\right) \propto \exp \left(\frac{-2 \pi \gamma_{0}\left(\rho^{2}\left(z_{r}\right)-\rho^{2}(z)\right)}{T}\right) .
$$

From Lawrencian geometries one could derive $A_{F F}$ :

$$
A_{F F}\left(z_{r}\right)=\frac{3 A}{4 r_{c n}^{3}} \int_{-l}^{z_{r}} \rho^{2}(\zeta) d \zeta
$$

Mass yield then determined using following relation:

$$
Y_{F M}\left(A_{F F} ; Z_{F S}, A_{F S}, E_{X}\right)=y\left(A_{F F}\right)+y\left(A-A_{F F}\right)
$$

\section{Calculation Procedure}

Procedure of calculation involves the creation of input file for TALYS. Fission simulation in TALYS are complex and require more adjustable parameter such as fission barrier and level density to obtain reasonable and better fission cross section and mass yield distribution.

1. Reaction of Am-241 (n,f)

Main parameter adjustment for this reaction including :

a. Incident energy range from $10^{-11} \mathrm{MeV}$ to 10 $\mathrm{MeV}$ b. Fission barrier parameter retrieved from experimental data.

c. Alternative fission barrier parameter from Sierk Model is used in case there is no experimental fission barrier.

d. Level density calculated using CTM model plus Fermi with level density parameter obtained using Ignatyuk formulation.

e. Triple humped fission barrier formalism is used in calculation.

f. Experimental fission barrier parameter is explicitly written in input file using values from other work (4).

g. Maximum vibrational band to be added to rotational coupling scheme in direct reaction set to 1 and maximum rotational band to be added to the same scheme is set to 4 .

2. Reaction of Th-232 (n,f)

a. Incident energy range from $10^{-11} \mathrm{MeV}$ to 20 $\mathrm{MeV}$.

b. Double humped fission barrier formalism is used in calculation.

c. Theoretical fission barrier parameters compiled in Mamdouth table is used in calculation.

d. Other parameter and reaction mechanism identical to the previous case.

3. Reaction of U-235 $(\mathrm{n}, \mathrm{f})$

All calculation procedure for this reaction are identical to reaction of Th-232 (n,f).

\section{Result and Analysis}

Figures 1, 3, 5 show the calculated fission cross sections of Am-241 (n,f), U-235 (n,f) and Th-232 (n,f) reaction, respectively. Fission cross section that obtained from TALYS prediction had worse agreement with experimental data from ENDF in case Am-241 (n,f) reaction. This could be caused by miss interpretation of fission barrier that used in calculation. For this case, one should try to use double humped barrier than triple humped one. Other cause can be yield from in-appropriate use of optical model parameter. The height of cross section curve is strongly related with the height and width of fission barrier, so adjustment process for this parameter is very required to get better agreement with experiment. In case for U-235 $(\mathrm{n}, \mathrm{f})$ reaction, double humped barrier is very appropriate to calculate fission cross section, the problem is to adjust all fission barrier parameter such as fission barrier height, transition states energy, barrier width and optical model parameter such as head band transition states, rotational band, moment of inertia, etc. For case three, that is Th-232 $(\mathrm{n}, \mathrm{f})$ reaction is similar to that of Am$241(\mathrm{n}, \mathrm{f})$. In this case, one should explicitly define the behavior of fission barrier to the input files, such as double-humped or triple humped and related parameter adjustment. Beside it, other parameter such as level density also has important role in describing transmission coefficient that would be used in cross 
section calculation. However, the first step to fix calculated fission cross section is to create properly fission barrier and optical model parameter according to the reaction mechanism.

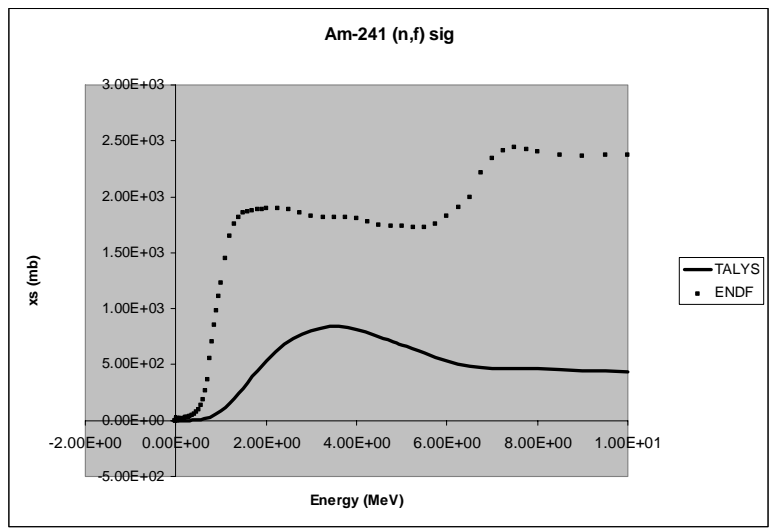

Figure 1. Fission cross section of Am-241 (n,f) reaction

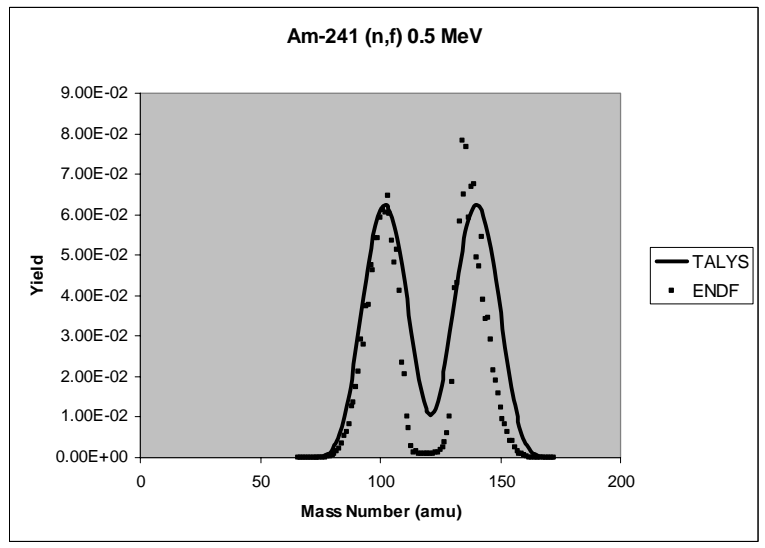

Figure 2. Mass yield distribution of Am-241 (n,f) reaction

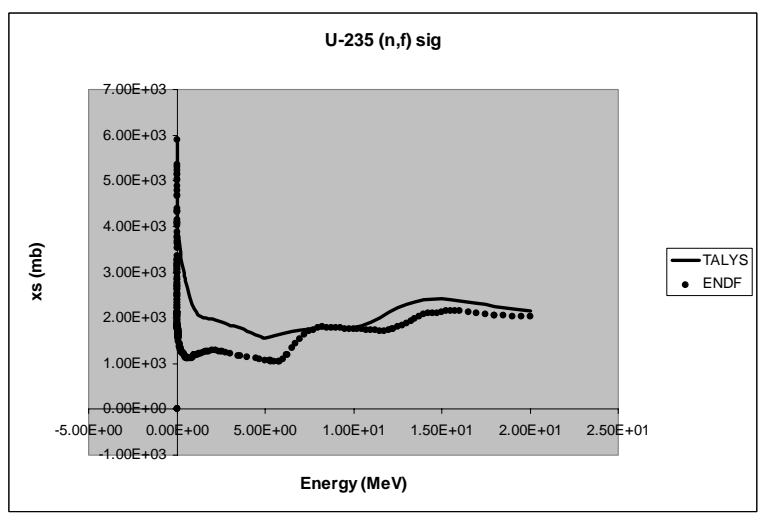

Figure 3. Fission cross section of U-235 (n,f)reaction

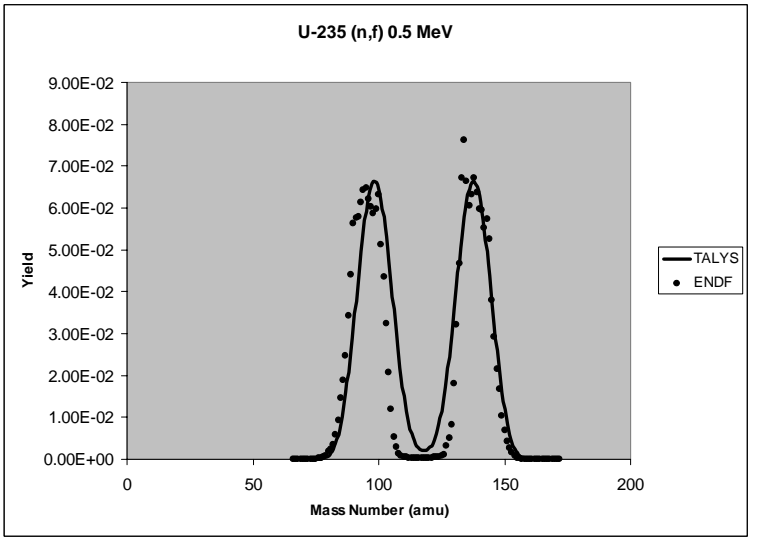

Figure 4. Mass yield distribution of U-235 (n,f) reaction

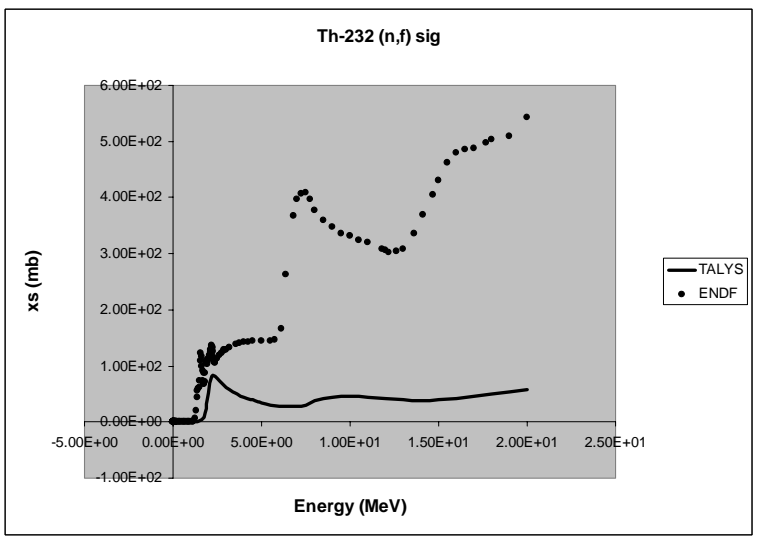

Figure 5. Fission cross section of Th-232 (n,f) reaction

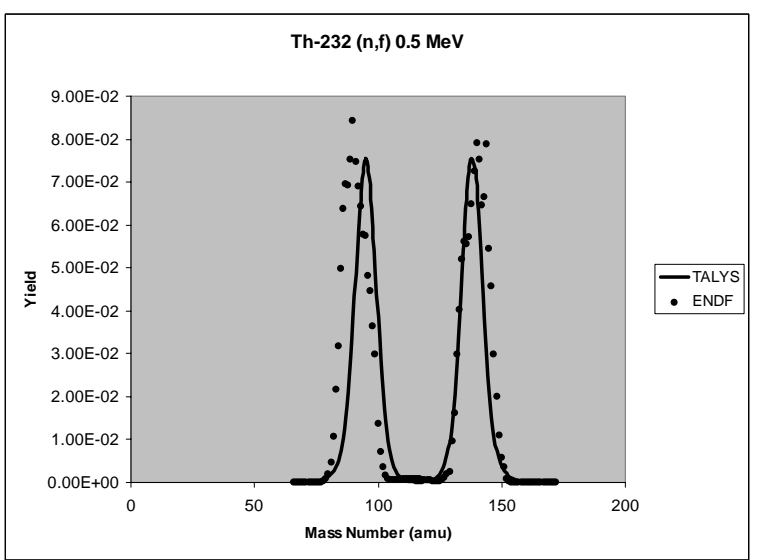

Figure 6. Mass yield distribution of Th-232 (n,f) reaction

Figures 2, 4, and 6 show the Mass yield distribution of Am-241 (n,f), U-235 (n,f) and Th-232 $(\mathrm{n}, \mathrm{f})$, correspondingly. Excitation energy grid that calculated from reaction mechanism is very important parameter to calculate yield of fission product besides neck parameter that provided from external library. In other hands, fission cross section per fissioning system is used to weight along with fission yield to form total 
fission yield. Inappropriate agreement between theoretical predictions using TALYS with experimental data from ENDF could be caused by several parameter described above. Neck parameter that used in Brosa model should be combined with correct excitation energy at deformed states to achieve better approximation at valley. Fission cross section that calculated from previous step had strong influence to mass distribution calculation.

In order to get better approximation, one should fix several parameter involved in fission barrier calculation and optical model used.

Curve shifting in mass distribution is likely caused by improper excitation binding energy and its value.

\section{Conclusion}

Calculation of some fission reaction parameters including Am-241 (n,f), Th-232 (n,f), and U-235 (n,f) reaction by employing TALYS code have been performed. Inappropriate agreement between theoretical predictions using TALYS with experimental data from ENDF could be caused by several parameters, such as fission barrier height, transition states energy, barrier width and optical model parameter such as head band transition states. Neck parameter that used in Brosa model should be combined with correct excitation energy at deformed states to achieve better approximation at valley. Fission cross section has strong influence to mass distribution calculation.

\section{References}

1. A.J. Koning, S. Hilaire and M.C. Duijvestijn, TALYS-1.0., Proceedings of the International Conference on Nuclear Data for Science and Technology - ND2007, May 22 - 27, 2007, Nice, France.

2. T. Ericson, Adv. Phys. 9, 425, 1960.

3. A.V. Ignatyuk, G.N. Smirenkin and A.S. Tishin, Sov. J. Nucl. Phys. 21:3, 25, 1975.

4. U. Brosa, Phys. Rev. C., 38, 4, 1998.

5. A. Gilbert and A.G.W. cameron, Can. J. Phys. 43, 1446, 1965.

6. A. J. Sierk, Phys. Rev. C., 33, 2039, 1986.

7. U. Brosa, S. Großmann and M“uller, Phys. Rep. 197, 167, 1990.

8. A. Mamdouh, J.M. Pearson, M. Rayet and F. Tondeur, Nucl. Phys. A679, 337, 2001. 